\title{
CHEWING ON GUM ALTERS THE EXPRESSION OF CONTAGIOUS YAWNING
}

\author{
Andrew C. Gallup ${ }^{1}$, Kaleigh Engert ${ }^{2}$ \\ ${ }^{1}$ Psychology Program, SUNY Polytechnic Institute, Utica, New York, USA \\ ${ }_{2}^{2}$ ssychology Department, SUNY College at Oneonta, Oneonta, New York, USA
}

a.c.gallup@gmail.com

\begin{abstract}
The motor action pattern of yawning enhances neurovascular circulation, which appears to function in cooling the brain and promoting adaptive behavioral outcomes linked with arousal and state change. Here, the authors tested whether chewing on gum, which induces similar mandibular contractions and temporarily increases alertness, alters the expression of yawning. Participants were assigned to either a control, mint, or gum condition and instructed to watch a contagious yawning stimulus in the laboratory. Yawning behavior was scored from video recordings. Findings showed that both sucking on a mint and chewing a piece of gum significantly inhibited contagion in these trials, as both manipulations diminished the overall rate and frequency of yawning in the sample. However, unlike sucking on a mint, chewing gum distinctly reduced and restricted the magnitude of the peak muscular contraction (i.e., the duration) of yawns and this measure correlated negatively with the frequency of chewing during testing. Overall, these findings add to our understanding of the physiology of yawning and provide directions for future research examining connections between yawning and cognitive performance.
\end{abstract}

Keywords: blood flow, brain temperature, cognitive performance, cortical arousal, mandibular contractions, swallowing. 


\section{INTRODUCTION}

Yawning is an involuntary and powerful stretching of the jaw with deep inspiration, followed by a temporary period of peak muscle contraction and passive closure of the jaw with expiration (Barbizet, 1958). Yawn-like motor action patterns with reflexive forms of mandibular stretching have been observed across vertebrates (Baenninger, 1987), and begin early on in intrauterine development (de Vries, Visser, \& Prechtl, 1982). These findings, coupled with psychological research showing hedonic properties to yawning (Provine, 1986) and the inability to achieve full yawns being perceived as frustrating (Walusinski, 2018), support the view that yawns evolved to serve an important biological function.

Numerous hypotheses regarding the physiological significance of yawning have been postulated (e.g., Smith, 1999), but few have garnered much empirical evidence (see Guggisberg, Mathis, Schnider, \& Hess, 2010; Gallup, 2011). For example, the still widely held notion that yawns function to increase and/or equilibrate oxygen levels in the blood was tested and falsified over 30 years ago (Provine, Tate, \& Geldmacher, 1987). More recent research suggests that a primary function of yawning is to enhance intracranial circulation (Walusinski, 2014), which, in turn, could promote arousal and state change (Provine, 1986; Baenninger, 1997; Provine, 2005) via brain cooling (Gallup \& Gallup, $2007 ; 2008)$. In support of this view, yawns tend to cluster around major transitions of activity (Baenninger, Binkley, \& Baenninger, 1996), important daily events (Baenninger 1987), and stressful situations and stimuli (Miller, Gallup, Vogel, \& Clark, 2010; Eldakar, Tartar, Garcia, Ramirez, Dauzonne \& Gallup, 2017). Moreover, yawns are linked with indicators of neurophysiological arousal (Sato-Suzuki, Kita, Oguri, \& Arita, 1998; SatoSuzuki, Kita, Seki, Oguri, \& Arita, 2002; Seki, Nakatani, Kita, Sato-Suzuki, Oguri, \& Arita, 2003; Kasuya, Murakami, Oshima, \& Dohi, 2005; Kita, Kubota, Yanagita, \& Motoki, 2008; but see Guggisberg, Mathis, Herrmann, \& Hess, 2007) and followed by significant decreases in brain and skull temperature (Shoup-Knox, Gallup, Gallup, \& McNay, 2010; Gallup \& Gallup, 2010; Equibar, Uribe, Cortes, Bautista, \& Gallup, 2017; Gallup, Herron, Militello, Swartwood, Cortes, \& Eguibar, 2017). Ultimately, these neurovascular changes may improve aspects of cognitive processing (Miller et al., 2010; Miller, Gallup, Vogel, \& Clark, 2012).

Further support for an important neurophysiological function to yawning comes from recent comparative studies examining how variation in the magnitude of this response (i.e., the duration of the peak musculature contraction) correlates with various neurological parameters across species. Using archived video footage to measure yawns from a diverse sample of mammals, interspecies yawn durations were found to be robustly correlated (coefficients $>0.9$ ) with measures of brain weight and cortical neuron number (Gallup, Church, \& Pelegrino, 2016). Similarly, species that displayed more variable yawn lengths tended to have larger and more complex brains. A follow-up study examining the relationship between yawn duration and brain size within a more restricted taxonomic scale (a family, Felidae) demonstrated a similarly robust association (Gallup, Crowe, \& Yanchus, 2017). Big cat species with larger brains, but not necessarily larger bodies, had longer average periods of mandibular gaping associated with their yawns. 
Since yawns appear to facilitate adaptive behavioral responses to inputs from the environment and internal states (reviewed by Baenninger, 1997), it is important to examine the variables that modulate this response. In particular, this study tested whether the manipulation of chewing gum altered the expression of contagious yawning among participants in a laboratory setting. The mandibular contractions associated with gum chewing have been associated with increased levels of both subjective (e.g., Scholey, Haskell, Robertson, Kennedy, Milne, \& Wetherell, 2009; Smith, 2009; Johnson, Jenks, Miles, Albert, \& Cox, 2011; Sketchley-Kaye, Jenks, Miles, \& Johnson, 2011; Allen \& Smith, 2012) and physiological measures of alertness (e.g., Smith, 2010; Allen, Jacob, \& Smith, 2014). In addition, mixed evidence shows that chewing gum improves performance on some cognitive tasks, such as working memory performance (for a review, see Allen \& Smith, 2011). The mechanisms contributing to these modulations in psychological processing are unclear, but likely include enhanced cerebral blood flow (Hasegawa, Ono, Hori, \& Nokubi, 2007), brain activation (Onozuka et al., 2002; Johnson et al., 2011) and glucose delivery (Stevens \& Tunney, 2004) produced by the mandibular contractions of chewing.

Given the similarities between the musculoskeletal involvement and the physiological and perceptual consequences associated with both yawning and gum chewing, we hypothesized that chewing gum would reduce the expression of yawning by inhibiting the mechanisms naturally triggering this response. Contagious yawning was used as a proxy for spontaneous (non-social) yawning since it can be reliably induced within the laboratory (Provine, 1986; Platek, Critton, Myers, \& Gallup, 2003). Moreover, recent studies have repeatedly shown that variables controlling the expression of spontaneous yawning also alter contagious yawning in the same fashion (Gallup \& Gallup, 2007; Massen, Dusch, Eldakar, \& Gallup, 2014; Eldakar, Dauzonne, Prilutzkaya, Garcia, Thadal, \& Gallup, 2015; Eldakar et al., 2017).

\section{METHODS}

A total of 127 ( 72 female; 19 unidentified) college-aged participants $(19.13 \pm 2.81$ years) were recruited through the psychology pool at a public four-year college in upstate New York during the spring and fall semesters of 2016. The experiment was conducted in accordance with approved human ethics guidelines, and all participants provided written informed consent prior to partaking in this study. Since participants were covertly recorded during testing, they were also provided with a video consent form at the end of the experiment. The local Institutional Review Board approved this research (\#2016-18).

Upon arriving at the laboratory, participants were escorted into an individual testing room and instructed to pay close attention to a 227-second contagious yawning stimulus on a computer monitor. This stimulus, played without sound, was from YouTube ("yawn-o-meter") and contained 50 consecutive clips of yawns (49 human, 1 dog). Prior to viewing this stimulus, rotating assignment designated participants to one of three 
conditions: (1) control ( $\mathrm{N}=43),(2)$ mint $(\mathrm{N}=42)$, and (3) gum chewing ( $\mathrm{N}=42)$. Participants in the control condition were asked to pay close attention to the video without further instruction, while those in the latter two groups were asked to either suck on a compressed sugar-free mint or chew a piece of sugar-free gum while viewing the stimulus. The mints and gum used in the study were from the same brand (Ice Breakers $^{\circledast}$ ) and very closely matched for flavor profile (spearmint) and ingredients. The ingredients for the mints were sorbitol and $2 \%$ or less of the following: maltodextrin, maltitol, natural and artificial flavor, aspartame, magnesium stearate, gum acacia, artificial color (blue 1, yellow 5), and soy lecithin. The ingredients for the gum included xylitol, gum base, maltitol syrup, mannitol, and $2 \%$ or less of the following: natural flavor and artificial flavor, gum acacia, maltodextrin, lecithin (soy), artificial color (blue 1 lake, yellow 5 lake, blue 1, yellow 5), aspartame, gelatin, acesulfame potassium, neotame, BHT to maintain freshness, and sucralose.

All participants were video recorded from a Logitech webcam positioned above the monitor while viewing the contagious yawning stimulus, from which a researcher scored yawning behavior and chew frequency (gum chewing condition only). Since previous research has shown that explicit surveillance or social presence cues inhibit contagious yawning in this setting (Gallup, Church, Miller, Risko, \& Kingstone, 2016), participants were not informed of the recording beforehand and there were no obvious visual cues (i.e., a light) indicating that the webcam was on. Participants also completed a short questionnaire afterwards, which included their age, time of testing, hours of sleep the night before, and whether they yawned or if they had the urge to yawn during the experiment. Webcam recordings were disrupted in 16 trials and one participant did not provide video consent, reducing the final sample size to 110 participants (control: $\mathrm{N}=39$; mint: $\mathrm{N}=34$; gum: $\mathrm{N}=37$ ).

Measures of interest included both yawn occurrence (yes/no) and yawn frequency, the latency to yawn following exposure to the video stimulus (in seconds), and yawn duration (to the nearest 0.01 second). Similar to Gallup et al. (2017), both average and maximum yawn duration were calculated along with variance (standard error) in this measure. All duration data were restricted to participants that yawned at least twice during the experiment to avoid potentially non-representative events from single yawns. There were no significant differences in the age or sex ratio of the participants, hours of sleep the night before, or testing times between conditions, and thus analyses consisted of Generalized Linear Models (GLMs) to test for differences between conditions across all yawn variables: occurrence (binary logistic), frequency (Poisson), latency (linear), and duration (linear). Spearman's rank correlations were used to assess the linear relationship between chew frequency and yawn duration within the gum chewing condition. Statistical analyses were conducted in SPSS v.25, and all data are represented in means and $95 \%$ confidence intervals. 


\section{RESULTS}

Forty-two participants (38.2\%) yawned at least once during the experiment. There was a significant effect of condition on yawn occurrence (Wald $X_{2}^{2}=10.709, p=0.005$; Fig. 1a), with a larger proportion of participants yawning in the control condition compared to both the mint condition $(p=0.008)$ and gum condition $(p=0.001)$. The overall frequency of yawning also varied significantly across conditions (Wald $X_{2}^{2}=49.852, p<$ 0.001 ; Fig. 1b). A greater number of yawns occurred in the control condition when compared to both the mint condition $(p<0.001)$ and gum condition $(p<0.001)$. In line with these findings, there was also a significant difference in the latency to yawn across conditions (Wald $X_{2}^{2}=9.800, p=0.007$ ), with participants in the control condition yawning sooner than those in both the mint condition $(p=0.007)$ and gum condition ( $p$ $=0.007)$.
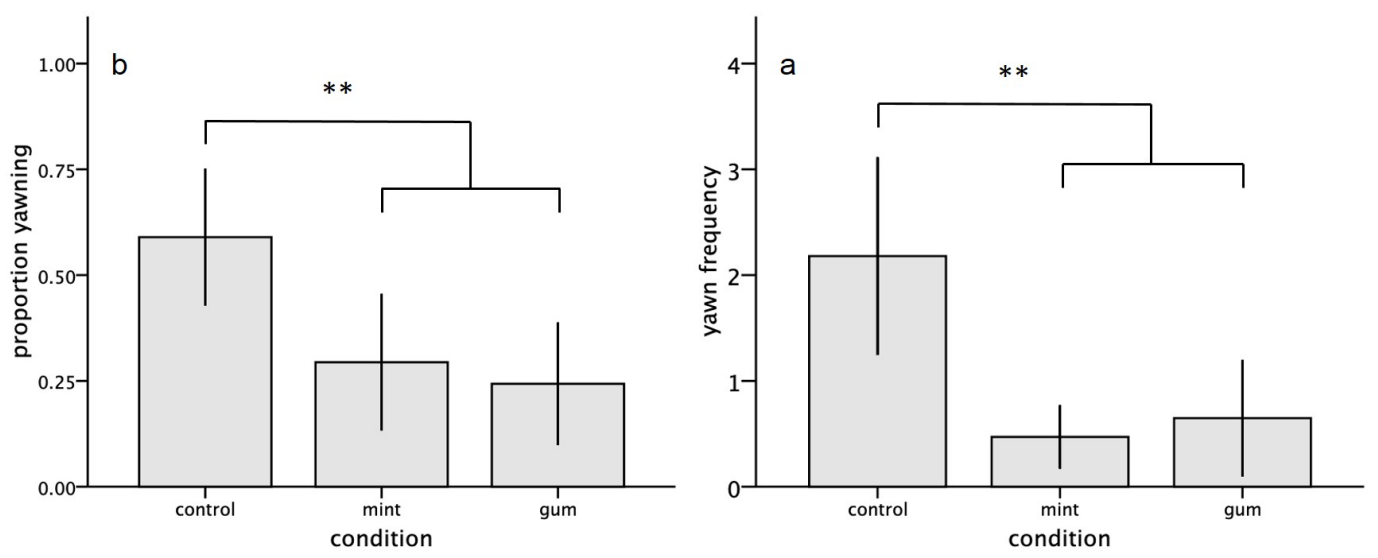

Figure 1: (a) Significantly fewer participants yawned and (b) significantly fewer yawns occurred in the mint and gum conditions compared to the control condition ${ }^{* *} p<$ $0.01)$.

Of the participants that yawned, $26(61.9 \%)$ did so at least twice during testing, permitting a comparison of yawn duration across conditions. Unlike the other yawning measures, average yawn duration did not vary significantly across the control, mint and gum conditions (Wald $X_{2}^{2}=3.086, p=0.214$ ). However, there was a significant effect of condition for maximum yawn duration (Wald $X_{2}^{2}=6.132, p=0.047$; Fig. 2a). Pairwise tests revealed that the maximum yawn durations observed during the control condition were longer than those in the gum condition $(p=0.013)$. In particular, the longest yawns in the control condition averaged $5.83 \mathrm{sec}$ in duration, while those in the gum condition were more than two seconds shorter $(3.52 \mathrm{sec})$. There was also a significant difference in the variance in yawn duration across conditions (Wald $X_{2}^{2}=6.454, p=0.040$ ), with the control condition showing greater variability compared to the gum condition ( $p=$ 0.021). Moreover, overall chew frequency in the gum condition was negatively correlated with both mean and maximum yawn duration $\left(\rho_{6}=-0.771, p=0.072\right.$; Fig. $2 b)$. 

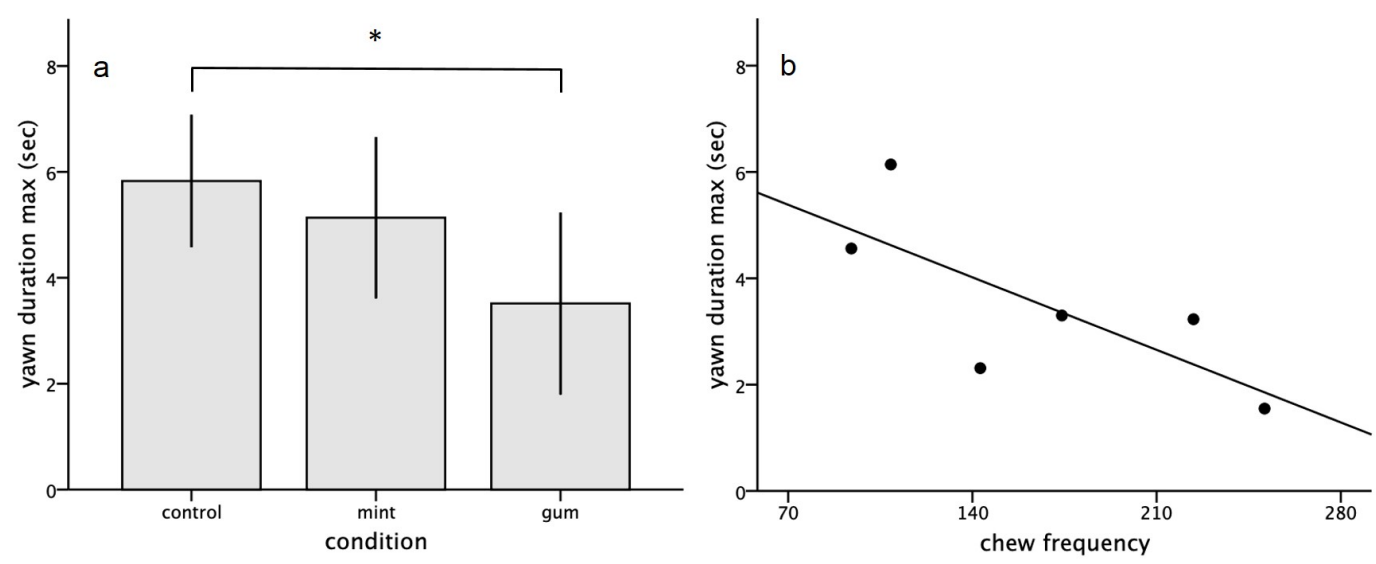

Figure 2: (a) The longest yawns recorded from participants in the gum condition were significantly shorter in duration compared to those in the control condition $\left({ }^{*} p<0.05\right)$ and (b) the chew frequency during testing was negatively correlated with this measure. Duration data were obtained from the 26 participants that yawned at least twice during the experiment (control: $\mathrm{N}=16$; mint: $\mathrm{N}=4$; gum: $\mathrm{N}=6$ ).

\section{DISCUSSION}

These findings are consistent with the hypothesis that mandibular contractions of chewing on gum would inhibit the expression of yawning. While nearly $60 \%$ of the participants yawned in the control condition, with an average of over two yawns each, participants that chewed gum were much less likely to yawn $(23.8 \%)$, yawned fewer times overall ( 0.71 yawns/participant), and required longer exposure to the stimulus prior to yawning at all. Moreover, chewing gum had distinct effects on the peak muscular contraction (i.e., duration) of this response. The longest yawns from participants in the gum condition were considerably shorter in duration compared to participant yawns in the control condition, and, as evidenced by the negative correlations with chew frequency, the mandibular contractions from chewing appeared to be driving this effect. The yawns in the gum condition were also less variable in duration. Sucking on a mint, however, did not significantly alter measures of yawn duration.

We suggest that the enhanced cerebral blood flow from chewing reduced the magnitude and restricted the expression of this response. Increased neurovascular circulation from chewing may also have similar brain cooling effects as yawning (ShoupKnox et al., 2010; Eguibar et al., 2017; Gallup et al., 2017), which could have contributed to the overall diminished response in this condition. Based on these findings, the authors speculate that these two behaviors may produce similar changes to cognitive processing. Although connections between gum chewing and cognition are mixed (Allen \& Smith, 2011), evidence suggests that working memory performance can be enhanced by this 
behavior (e.g., Wilkinson, Scholey, \& Wesnes, 2002; Stevens \& Tunney, 2004; Hirano et al., 2008). This is noteworthy given previous research has suggested that, by cooling the brain, yawns could enhance mental processing efficiency on tasks evoking working memory (i.e., vigilance) (Gallup \& Gallup, 2007). Moreover, comparative studies have shown that yawning increases under conditions that trigger enhanced vigilance to environmental stimuli (Miller et al., 2010; 2012). Therefore, future research could examine whether inducing yawns improves performance on working memory tasks in a similar fashion to chewing gum. Given the distinct circadian pattern of this behavior, occurring primarily after waking and prior to sleeping (Provine, Hamernik, \& Curchack, 1987; Baenninger et al., 1996), it would be particularly informative to compare differences in cognition following yawning in the morning and evening hours.

The current study also revealed the unexpected finding that sucking on a mint significantly increased the latency to yawn and reduced the overall occurrence and frequency of yawn contagion during testing. The authors speculate that enhanced nasal breathing, which is a natural brain cooling mechanism (Mariak, White, Lewko, Lyson, \& Piekarski, 1999) and has been shown to reduce contagious yawning in the laboratory (Gallup \& Gallup 2007), contributed to this effect. Sucking on mints naturally alters breathing patterns, by reducing oral inspiration and expiration and promoting exclusive nasal breathing. Furthermore, it has been suggested that yawning and swallowing share common neuroanatomical and physiological pathways (Ertekin, Bulbul, Uludag, Tiftikcioglu, Arici, \& Gurgor, 2015), and thus increased swallowing in the mint condition may have inhibited yawns. While overall swallowing patterns were not obtainable from the videos in this study, we suggest that further research could examine how the manipulation of swallowing alters yawning.

Overall, this report provides novel findings that add to our understanding of the mechanisms controlling yawning. Given the similarities between the physiological and psychological consequences of yawning and chewing on gum, as well as research showing positive associations between masticatory performance and cognition (Teixeira et al., 2014), the link between these behaviors warrants further investigation. Although both yawning and chewing on gum can be considered inappropriate in social settings, these behaviors appear to share intrinsic properties that may be associated with beneficial psychological and perceptual changes. 


\section{COMPLIANCE WITH ETHICAL STANDARDS}

Funding: ACG received funding from the College of Arts \& Sciences at SUNY Polytechnic Institute.

Conflict of Interest: The authors declare they have no conflicts of interest.

Ethics Approval: This article included human subjects research that was approved by a local ethics board, and written informed consent was provided by all participants in this study.

Data Availability: The dataset from the current study is available by request from the corresponding author.

\section{ACKNOWLEDGMENTS}

We are grateful to Michael Yanchus, Margret Potrikus and Ryan Butler for helping collect and collate data for this study. We are also grateful for fruitful discussion and feedback on this project from members of the Evolutionary Psychology Lab at SUNY Oneonta from 2016-2017.

\section{REFERENCES}

Allen, A. P., Jacob, T. J., \& Smith, A. P. (2014). Effects and after-effects of chewing gum on vigilance, heart rate, EEG and mood. Physiology \& Behavior, 133, 244-251. DOI

Allen, A. P., \& Smith, A. P. (2011). A review of the evidence that chewing gum affects stress, alertness and cognition. Journal of Behavioral and Neuroscience Research, 9(1), 7-23.

Allen, A. P., \& Smith, A. P. (2012). Effects of chewing gum and time-on-task on alertness and attention. Nutritional Neuroscience, 15(4), 176-185.

Barbizet, J. (1958). Yawning. Journal of Neurology, Neurosurgery, and Psychiatry, 21(3), 203. DOI

Baenninger, R. (1987). Some comparative aspects of yawning in Betta splendens, Homo sapiens, Panthera leo, and Papio sphinx. Journal of Comparative Psychology, 101(4), 349-354. $\underline{\mathrm{DOI}}$

Baenninger, R. (1997). On yawning and its functions. Psychonomic Bulletin \& Review, 4(2), 198-207. DOI

Baenninger, R., Binkley, S., \& Baenninger, M. (1996). Field observations of yawning and activity in humans. Physiology \& Behavior, 59(3), 421-425. DOI 
De Vries, J. I., Visser, G. H., \& Prechtl, H. F. (1982). The emergence of fetal behaviour. I. Qualitative aspects. Early Human Development, 7(4), 301-322. DOI

Eguibar, J. R., Uribe, C. A., Cortes, C., Bautista, A., \& Gallup, A. C. (2017). Yawning reduces facial temperature in the high-yawning subline of Sprague-Dawley rats. $B M C$ Neuroscience, 18(1), 3. DOI

Eldakar, O. T., Dauzonne, M., Prilutzkaya, Y., Garcia, D., Thadal, C., \& Gallup, A. C. (2015). Temperature-dependent variation in self-reported contagious yawning. Adaptive Human Behavior and Physiology, 1(4), 460-466. DOI

Eldakar, O. T., Tartar, J. L., Garcia, D., Ramirez, V., Dauzonne, M., Armani, Y., \& Gallup, A. C. (2017). Acute physical stress modulates the temporal expression of self-reported contagious yawning in humans. Adaptive Human Behavior and Physiology, 3(2), 156-170. $\underline{\mathrm{DOI}}$

Ertekin, C., Bulbul, N. G., Uludag, I. F., Tiftikcioglu, B. I., Arici, S., \& Gurgor, N. (2015). Electrophysiological association of spontaneous yawning and swallowing. Experimental Brain Research, 233(7), 2073-2080. DOI

Gallup, A. C. (2011). Why do we yawn? Primitive versus derived features. Neuroscience \& Biobehavioral Reviews, 35(3), 765-769. DOI

Gallup, A. C., \& Gallup Jr, G. G. (2007). Yawning as a brain cooling mechanism: nasal breathing and forehead cooling diminish the incidence of contagious yawning. Evolutionary Psychology, 5(1), 92-101. DOI

Gallup, A. C., \& Gallup Jr, G. G. (2008). Yawning and thermoregulation. Physiology \& Behavior, 95(1-2), 10-16. DOI

Gallup, G. G., \& Gallup, A. C. (2010). Excessive yawning and thermoregulation: two case histories of chronic, debilitating bouts of yawning. Sleep and Breathing, 14(2), 157-159. $\underline{\mathrm{DOI}}$

Gallup, A. C., Church, A. M., \& Pelegrino, A. J. (2016). Yawn duration predicts brain weight and cortical neuron number in mammals. Biology Letters, 12(10), 20160545. DOI

Gallup, A., Church, A. M., Miller, H., Risko, E. F., \& Kingstone, A. (2016). Social presence diminishes contagious yawning in the laboratory. Scientific Reports, 6(1), 25045. DOI

Gallup, A. C., Herron, E., Militello, J., Swartwood, L., Cortes, C., \& Eguibar, J. R. (2017). Thermal imaging reveals sizable shifts in facial temperature surrounding yawning in budgerigars (Melopsittacus undulatus). Temperature, 4(4), 429-435. DOI

Gallup, A. C., Crowe, B., \& Yanchus, M. (2017). Yawn duration predicts brain volumes in wild cats (Felidae). International Journal of Comparative Psychology, 30.

Guggisberg, A. G., Mathis, J., Herrmann, U. S., \& Hess, C. W. (2007). The functional relationship between yawning and vigilance. Behavioural Brain Research, 179(1), 159-166. DOI

Guggisberg, A. G., Mathis, J., Schnider, A., \& Hess, C. W. (2010). Why do we yawn?. Neuroscience \& Biobehavioral Reviews, 34(8), 1267-1276. DOI

Hasegawa, Y., Ono, T., Hori, K., \& Nokubi, T. (2007). Influence of human jaw movement on cerebral blood flow. Journal of Dental Research, 86(1), 64-68. DOI

Hirano, Y., Obata, T., Kashikura, K., Nonaka, H., Tachibana, A., Ikehira, H., \& Onozuka, M. (2008). Effects of chewing in working memory processing. Neuroscience Letters, 436(2), 189-192. DOI

Johnson, A. J., Jenks, R., Miles, C., Albert, M., \& Cox, M. (2011). Chewing gum moderates multi-task induced shifts in stress, mood, and alertness. A re-examination. Appetite, 56(2), 408-411. DOI 
Kasuya, Y., Murakami, T., Oshima, T., \& Dohi, S. (2005). Does yawning represent a transient arousal-shift during intravenous induction of general anesthesia?. Anesthesia \& Analgesia, 101(2), 382-384. DOI

Kita, I., Kubota, N., Yanagita, S., \& Motoki, C. (2008). Intracerebroventricular administration of corticotropin-releasing factor antagonist attenuates arousal response accompanied by yawning behavior in rats. Neuroscience Letters, 433(3), 205-208. DOI

Mariak, Z., White, M. D., Lewko, J., Lyson, T., \& Piekarski, P. (1999). Direct cooling of the human brain by heat loss from the upper respiratory tract. Journal of Applied Physiology, 87(5), 1609-1613. DOI

Massen, J. J., Dusch, K., Eldakar, O. T., \& Gallup, A. C. (2014). A thermal window for yawning in humans: yawning as a brain cooling mechanism. Physiology \& Behavior, 130, 145-148. $\underline{\text { DOI }}$

Miller, M. L., Gallup, A. C., Vogel, A. R., \& Clark, A. B. (2010). Handling stress initially inhibits, but then potentiates yawning in budgerigars (Melopsittacus undulatus). Animal Behaviour, 80(4), 615-619. DOI

Miller, M. L., Gallup, A. C., Vogel, A. R., \& Clark, A. B. (2012). Auditory disturbances promote temporal clustering of yawning and stretching in small groups of budgerigars (Melopsittacus undulatus). Journal of Comparative Psychology, 126(3), 324. DOI

Onozuka, M., Fujita, M., Watanabe, K., Hirano, Y., Niwa, M., Nishiyama, K., \& Saito, S. (2002). Mapping brain region activity during chewing: a functional magnetic resonance imaging study. Journal of Dental Research, 81(11), 743-746. DOI

Platek, S. M., Critton, S. R., Myers, T. E., \& Gallup Jr, G. G. (2003). Contagious yawning: the role of self-awareness and mental state attribution. Cognitive Brain Research, 17(2), 223-227. DOI

Provine, R. R. (1986). Yawning as a stereotyped action pattern and releasing stimulus. Ethology, 72(2), 109-122. DOI

Provine, R. R. (2005). Yawning: the yawn is primal, unstoppable and contagious, revealing the evolutionary and neural basis of empathy and unconscious behavior. American Scientist, 93(6), 532-539.

Provine, R. R., Tate, B. C., \& Geldmacher, L. L. (1987a). Yawning: no effect of 3-5\% CO2, 100\% O2, and exercise. Behavioral and Neural Biology, 48(3), 382-393. DOI

Provine, R. R., Hamernik, H. B., \& Curchack, B. C. (1987b). Yawning: relation to sleeping and stretching in humans. Ethology, 76(2), 152-160. DOI

Sato-Suzuki, I., Kita, I., Oguri, M., \& Arita, H. (1998). Stereotyped yawning responses induced by electrical and chemical stimulation of paraventricular nucleus of the rat. Journal of Neurophysiology, 80(5), 2765-2775. DOI

Sato-Suzuki, I., Kita, I., Seki, Y., Oguri, M., \& Arita, H. (2002). Cortical arousal induced by microinjection of orexins into the paraventricular nucleus of the rat. Behavioural Brain Research, 128(2), 169-177. DOI

Scholey, A., Haskell, C., Robertson, B., Kennedy, D., Milne, A., \& Wetherell, M. (2009). Chewing gum alleviates negative mood and reduces cortisol during acute laboratory psychological stress. Physiology \& Behavior, 97(3-4), 304-312. DOI

Seki, Y., Nakatani, Y., Kita, I., Sato-Suzuki, I., Oguri, M., \& Arita, H. (2003). Light induces cortical activation and yawning in rats. Behavioural Brain Research, 140(1-2), 65-73. $\underline{\mathrm{DOI}}$

Shoup-Knox, M. L., Gallup, A. C., Gallup, G., \& McNay, E. C. (2010). Yawning and stretching predict brain temperature changes in rats: support for the thermoregulatory hypothesis. Frontiers in Evolutionary Neuroscience, 2, 108. DOI 
Sketchley-Kaye, K., Jenks, R., Miles, C., \& Johnson, A. J. (2011). Chewing gum modifies state anxiety and alertness under conditions of social stress. Nutritional Neuroscience, 14(6), 237-242. DOI

Smith, A. (2009). Effects of chewing gum on mood, learning, memory and performance of an intelligence test. Nutritional Neuroscience, 12(2), 81-88. DOI

Smith, A. (2010). Effects of chewing gum on cognitive function, mood and physiology in stressed and non-stressed volunteers. Nutritional Neuroscience, 13(1), 7-16. DOI

Smith, E. O. (1999). Yawning: an evolutionary perspective. Human Evolution, 14(3), 191-198. $\underline{\mathrm{DOI}}$

Stephens, R., \& Tunney, R. J. (2004). Role of glucose in chewing gum-related facilitation of cognitive function. Appetite, 43(2), 211-213. DOI

Teixeira, F. B., Fernandes, L. D. M. P., Noronha, P. A. T., dos Santos, M. A. R., Gomes-Leal, W., Maia, C. D. S. F., \& Lima, R. R. (2014). Masticatory deficiency as a risk factor for cognitive dysfunction. International Journal of Medical Sciences, 11(2), 209-214. DOI

Walusinski, O. (2014). How yawning switches the default-mode network to the attentional network by activating the cerebrospinal fluid flow. Clinical Anatomy, 27(2), 201-209. $\underline{\mathrm{DOI}}$

Walusinski, O. (2018). Pathological Yawning, Laughing and Crying. In Neurologic-Psychiatric Syndromes in Focus-Part I (Vol. 41, pp. 40-49). Karger Publishers. DOI

Wilkinson, L., Scholey, A., \& Wesnes, K. (2002). Chewing gum selectively improves aspects of memory in healthy volunteers. Appetite, 38(3), 235-236. DOI 